\title{
Network structure of polyfluorene sheets as a function of alkyl side chain length
}

\author{
M. Knaapila,,${ }^{1, *}$ D. W. Brightt, ${ }^{2}$ R. Stepanyan, ${ }^{3}$ M. Torkkeli, ${ }^{4}$ L. Almásy,${ }^{5,6}$ R. Schweins, ${ }^{7}$ U. Vainio, ${ }^{8}$ \\ E. Preis, ${ }^{9}$ F. Galbrecht, ${ }^{9}$ U. Scherf, ${ }^{9}$ and A. P. Monkman ${ }^{2}$ \\ ${ }^{1}$ Physics Department, Institute for Energy Technology, NO-2027 Kjeller, Norway \\ ${ }^{2}$ Department of Physics, University of Durham, DH1 3LE Durham, United Kingdom \\ ${ }^{3}$ Materials Science Centre, DSM Research, NL-6160 MD Geleen, The Netherlands \\ ${ }^{4}$ Department of Physics, FI-00014 University of Helsinki, Finland \\ ${ }^{5}$ Research Institute for Solid State Physics and Optics, Budapest-1525, Hungary \\ ${ }^{6}$ Laboratory for Neutron Scattering, PSI, CH-5232 Villigen, Switzerland \\ ${ }^{7}$ Institut Laue-Langevin, DS / LSS group, FR-38042 Grenoble CEDEX 9, France \\ ${ }^{8}$ HASYLAB am DESY, DE-22607 Hamburg, Germany \\ ${ }^{9}$ Fachbereich Chemie, Bergische Universität Wuppertal, DE-42097 Wuppertal, Germany
}

(Received 2 February 2011; published 19 May 2011)

\begin{abstract}
The formation of self-organized structures in poly(9,9-di- $n$-alkylfluorene)s $\sim 1$ vol \% methylcyclohexane $(\mathrm{MCH})$ and deuterated $\mathrm{MCH}\left(\mathrm{MCH}-d_{14}\right)$ solutions was studied at room temperature using neutron and $\mathrm{x}$-ray scattering (with the overall $q$ range of $0.00058-4.29 \AA^{-1}$ ) and optical spectroscopy. The number of side chain carbons $(N)$ ranged from 6 to 10. The phase behavior was rationalized in terms of polymer overlap, cross-link density, and blending rules. For $N=6-9$, the system contains isotropic areas and lyotropic areas where sheetlike assemblies (lateral size of $>400 \AA$ ) and free polymer chains form ribbonlike agglomerates (characteristic dimension of $>1500 \AA$ ) leading to a gel-like appearance of the solutions. The ribbons are largely packed together with surface fractal characteristics for $N=6-7$ but become open networklike structures with mass fractal characteristics for $N=8-9$, until the system goes through a transition to an isotropic phase of overlapping rodlike polymers for $N=10$. The polymer order within sheets varies allowing classification for loose membranes and ordered sheets, including the so-called $\beta$ phase. The polymers within the ordered sheets have restricted motion for $N=6-7$ but more freedom to vibrate for $N=8-9$. The nodes in the ribbon network are suggested to contain ordered sheets cross-linking the ribbons together, while the nodes in the isotropic phase appear as weak density fluctuations cross-linking individual chains together. The tendencies for macrophase separation and the formation of non beta sheets decrease while the proportion of free chains increases with increasing $N$. The fraction of $\beta$ phase varies nonlinearly, reaching its maximum at $N=8$.
\end{abstract}

DOI: 10.1103/PhysRevE.83.051803

PACS number(s): 61.25.he, 82.70.Gg, 87.16.dr, 61.05.fg

\section{INTRODUCTION}

Structure and spatial fluctuations of gels of flexible polymers are well-understood topics both from theoretical [1] and experimental [2] points of view. Less attention has been placed on the gels of $\pi$-conjugated hairy-rod-type polymers with stiff backbones and flexible side chains, although the solid state structure of their major classes are well described [3]. The demarcation lines between solution, gel, and macrophase separation of $\pi$-conjugated polymers are not well defined and the terms are often considered as phenomenological. What is referred to as a solution in an experiment is not necessarily an isotropic mixture of polymers but can contain various kinds of assemblies. The ability to solution process thin film devices is an advantage of $\pi$-conjugated polymers over oligomers [4], and the polymer morphology (and thus the device performance in the solid state) is influenced by the possible polymer assemblies in the solution, as shown for instance for poly(9,9-dioctylfluorene-co-benzothiadiazole) by Banach et al. [5]. Identifying the structure of such assemblies is therefore important not only from the fundamental point of view, for studying a balance between solution, gel, and macrophase separation, but also from a device processing

*matti.knaapila@ife.no point of view. Selective use of solvents is a tool for top-down nanotechnology used, for example, to form ringlike nano- and dotlike microscale formations of poly(9,9-didecylfluorene) (PF10) [6]. These phenomena are also driven by aggregation tendencies.

Amongst the $\pi$-conjugated polymers poly(2,7-fluorene)s (PFs) $[7,8]$ are well-known blue emitters [9] widely employed in solution-processed polymer electronics [10]. PFs can be synthesized in increasingly complicated chemical structures [11] but much can be learned already by considering archetypical PFs with linear alkyl side chains [12]. A prime example is poly(9,9-dioctylfluorene) (PF8) $[13,14]$ which shows crystalline $\alpha$ and $\alpha^{\prime}$ phases [15,16], noncrystalline $\beta$, nematic, and amorphous phases [17], and as a $g$ phase [18] in the solid state. The $\beta$ phase $[19,20]$ has a mesomorphic structure [21] associated with narrow linewidths in absorption, prompt, and delayed fluorescence, phosphorescence, and photoinduced triplet absorption spectra [22]. The $\beta$ phase seems to require the presence of solvent molecules while the others are "true" solid state phases. This solid state framework represents the base for studying PF solutions and gels. A logical first step towards understanding increased structural complexity is to change the side chain length that alters the solid state structure which has been shown so far for poly(9,9-dihexylfluorene) (PF6) [23]. In our notation this factor is characterized by the number of side chain beads, $N$. 
Kitts and Vanden Bout [24] studied PF8 and demonstrated how various solvents have significant effects on the optical characteristics matching the twisted and planar polymer chains, with the latter being favored by poor solvents. PF8 in toluene or methylcyclohexane $(\mathrm{MCH})[25,26]$ is an illustrative example of how polymer aggregation is enhanced in the poorer solvent $(\mathrm{MCH})$ or by increasing polymer concentration, decreasing temperature, or aging. In toluene, PF8 is dissolved locally down to the single polymer level when concentration is sufficiently low $(\leqslant 10 \mathrm{mg} / \mathrm{mL})$ at room temperature but it assembles into a network with associated domains, if the concentration is increased $(\geqslant 30 \mathrm{mg} / \mathrm{mL})$ [27,28] or if the system is cooled down [29], as comprehensively shown by Chen and co-workers. Stiff chains tend to pack in a unidirectional fashion within the polymer assemblies justifying the term segmental alignment [27]. In $\mathrm{MCH}$, the sheetlike assemblies appear with decreasing temperature [26] or by room temperature aging driven by macrophase separation, which leads to coexistence of an isotropic phase and an assembly-rich phase with local heterogeneities [30]. The gel-like appearance and optical characteristics of the $\beta$ phase follow from the polymer assemblies and a sharp transition back to the isotropic phase is observed upon heating.

The picture becomes rather more complicated when $N$ is varied, in our example from $N=6$ to $N=10$, i.e., from PF6 via poly(9,9-diheptylfluorene) (PF7), PF8, and poly(9,9-dinonylfluorene) (PF9) to PF10. The assemblies are favored by decreasing $N$ both in toluene [31] and MCH [32]. If the assemblies are assumed to be quasi-two-dimensional membranes, the isotropic phase transition temperature $\left(T_{\mathrm{mem}}^{*}\right)$ for the membrane generally increases with decreasing $N$ [33] and is unattainable for PF6. At room temperature, the optical characteristics matching those of the PF8 $\beta$ phase are found for PF7 and PF9 in MCH both at high ( $\sim 1$ vol \%) [32] and low $(\sim 0.002$ vol \%) [34] concentrations, while the same is seen for PF10 in thin films [35], with its thermal dependency following the lower $T_{\mathrm{mem}}^{*}$ [33].

The presence of sheetlike assemblies [26] or membranes [33] in PFs mixed with $\mathrm{MCH}$ (or $\mathrm{PF}_{N}-\mathrm{MCH}$ ) is well established and discussed in terms of their internal structure [32] and macrophase separation [30], thermal behavior [30,32], and the existence of $\beta$ phase [25,34,35]. Attention should also be placed on the density fluctuations or aggregation of the sheetlike assemblies. This system may be called a lyotropic phase with solvent coexistence [33] and the fluctuations called "heterogeneities in the liquid crystalline domains" as suggested by Chen et al. [30]. We should also be aware of the coexistence of these density fluctuations and assemblies themselves and the coexisting assembly types, i.e., membranes, $\beta$ sheets, and free chains [33].

In this paper we show how $\mathrm{PF}_{N}-\mathrm{MCH}$ behaves with varying $N$ in terms of larger agglomerates of sheetlike polymer assemblies. We studied this system using small-angle neutron scattering (SANS) combined with small- and wide-angle X-ray scattering (SAXS and WAXS) and optical spectroscopy. $\mathrm{PF}_{N}-\mathrm{MCH}$ contains isotropic and assembly-rich phases. We suggest that all assemblies are relatively stiff and their size does not change much with increasing $N$ but they form larger ribbonlike agglomerates, which form networks with greater openness with increasing $N$. We also show how the relative proportion of each assembly type changes with $N$. This leads to a more complete picture of the structural levels for each $N$ and may be rationalized in terms of polymer overlap, cross-link density, and blending rules.

\section{THEORY}

\section{A. Polymer overlap}

Our previous work identified sheetlike assemblies of linear side chain PFs that can either be "loose" or "ordered" [33]. The majority of these assemblies appeared as loose bilayers and were denoted as "membranes." PFs can also appear as locally free chains that join to the domains denoted as "segmental alignment" over longer length scales at high concentrations [27]. The intent of our work is to study fluctuations of these assemblies with increasing numbers of side chain beads $N$.

The prerequisite for both the agglomerates of polymer assemblies and presumably, gel formation, is polymer overlap as discussed thoroughly by Chen et al. [29]. Following the arguments of Ying and Chu [36] and Graessley [37], the polymer overlap concentration is defined as the polymer mass per unit volume:

$$
c^{*} \sim M_{n} / N_{A}\left(2 R_{g}\right)^{3},
$$

where $R_{g}$ is the radius of gyration of a single polymer chain.

The radius of gyration may be estimated from the KratkyPorod equation for wormlike polymers

$$
R_{g}^{2} \sim l_{p} l_{0} M_{n} / 3 M_{0},
$$

where $l_{p}$ and $l_{0}$ are the persistence length and the length of the repeat unit, and $M_{n}$ and $M_{0}$ are the number-averaged molecular weight of the polymer and the weight of the monomer unit, respectively.

\section{B. Agglomeration of polyfluorene sheets}

The $\mathrm{PF}_{N}-\mathrm{MCH}$ system with sheetlike polymer assemblies consists of domains with pure solvent and domains where both polymer (in its various forms) and solvent are present. This means that macrophase separation emerges but the system is not demixed on the large scale, possibly due to high viscosity.

The stiff sheetlike assemblies are expected to grow until they form continuous ribbonlike agglomerates. The sheets are thus building blocks of the ribbons and appear rigid and with similar shapes with increasing $N$. The ribbons are flexible, appearing as different shapes with increasing $N$ (vide infra). The ribbons are expected to form a networklike structure and make the system appear milky. We aim to understand this structure as a function of $N$ at fixed temperature. To answer this question we give the following arguments:

(1) As the viscosity in the ribbon phase is significantly higher than that in the isotropic phase, the ribbons may be cross-linked together. These cross-links are thus links between ribbons and not between individual polymers.

(2) The distance between cross-links and the distance between ribbons are not independent but they scale similarly with $N$.

(3) Cross-links must show higher order than the ribbon assemblies on average. To form an ordered node, two ribbons 
must come into contact and "cocrystallize." This means that the probability for cross-link is

$$
p(\text { cross-link })=p(\text { meeting }) p(\text { ordered }),
$$

where $p$ (meeting) is the probability that two ribbons meet each other and $p$ (ordered) is the probability that at the point where the two ribbons connect, a weakly ordered or crystalline structure is formed.

The chance of meeting depends on the square of the concentration of the ribbons

$$
p(\text { meeting }) \sim c_{R}^{2},
$$

where $c_{R}$ is the ribbon concentration. As polymers may also appear as free chains, $c_{R}<c$, where the overall polymer concentration $c$ (the polymer mass per solvent volume in a sample) is the same for all $N$.

The order in the ribbons may be attributed to the $\beta$ phase known to exist in PF8. This weakly ordered structure forms in the presence of solvent while the other ordered "true crystalline" phases of PF8 exist in the solid state. The $\beta$ phase involves a planarization of the backbone $[13,14]$ which may be driven by the external environment. Bright et al. [35] studied the optical trace of the $\beta$ phase in $\mathrm{MCH}$ for $N=6-10$ and proposed that its formation is balanced between two factors. The tendency for polymer assemblies decreases with increasing $N$, but the van der Waals bond energy available to overcome the steric repulsion and planarize the backbone increases with increasing $N$. This leads the proportion of $\beta$ phase to reach a peak at $N=8$. Assuming that the nodes contain a weakly ordered $\beta$ phase, we say

$$
p(\beta) \text { has a maximum at } N=8 \text {. }
$$

The order in the ribbons may be attributed to any undefined ordered structure with a probability of $p$ (cryst.). From experiments we know that the $\mathrm{PF}_{N}-\mathrm{MCH}$ system [32] shows x-ray diffraction peaks arising from the ordered structure at room temperature for $N=6-9$. As the peak intensity and width do not significantly vary with $N$, we assume that $p$ (cryst.) is constant or decreases with increasing $N$.

The exact nature of cross-links is not significant on larger length scales. We may therefore write

$$
p(\text { ordered })=p(\beta)+p(\text { cryst. }) .
$$

We will next illustrate the factors influencing mixing of polymer ribbons in solution and how these might depend on $p$ (cross-link) as a function of $N$. For simplicity we consider ribbon assemblies as if they were polymer chains and try to define an "effective" interaction parameter $\bar{\chi}$ between the ribbons and solvent. This parameter describes the interaction between mixed entities and can be used to describe free energy of mixing as a function of composition. The polymer interaction may be described in various ways (see, for example, Ref. [38]) but a blending rule is generally used. In its simplest form this yields

$$
\begin{aligned}
\bar{\chi} & =y \chi_{S A}+(1-y) \chi_{S R}-y(1-y) \chi_{A R} \\
& =\chi_{S R}-y\left[\chi_{S R}-\chi_{S A}+(1-y) \chi_{A R}\right],
\end{aligned}
$$

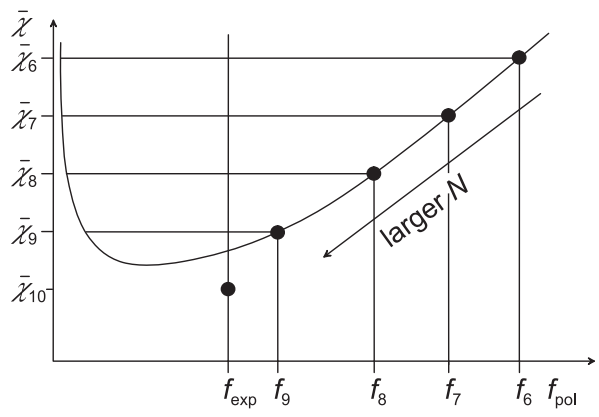

FIG. 1. Illustrations of the phase equilibria in the ribbonlike agglomerates of $\mathrm{PF}_{N}-\mathrm{MCH}$. The biphasic region within the "cup" yields almost pure solvent (volume fraction of membranes $f \sim 0$ ) on the right and the solution of the ribbons on the left from the minimum. The arrow shows that the polymer concentration in the ribbon-rich domains is expected to grow with increasing $N$. At a certain side chain length ( $N=10$ in our case) no phase separation occurs.

where a bar denotes effective and $y$ is the volume fraction of alkyl chains in the hairy-rod polymer. The subindices are " $S$ " = solvent, " $A$ " = alkyl, and " $R$ " = rod.

$\mathrm{MCH}$ is a poor solvent for both the side chains and polymer backbones so both $S A$ and $S R$ interactions are significant. Nevertheless, aliphatic $\mathrm{MCH}$ is still a poorer solvent for the aromatic polymer backbone $\left(0<\chi_{S A}<\chi_{S R}\right)$ and increasing $N$ increases polymer solubility in $\mathrm{MCH}$. Therefore, we assume that the $S R$ interaction is dominant and simplify

$$
\bar{\chi} \sim \frac{v}{v_{0} N+v} \chi_{S R},
$$

where $v$ is the volume of the repeat unit of the rod, $v_{0}$ is the volume of one side chain bead. Increasing $N$ gives lower $\bar{\chi}$ and this may lead to a solvent-polymer phase diagram, illustrated in Fig. 1. This diagram shows $\bar{\chi}$ against the limiting volume fraction of membranes $(f)$ for $N=6-10$. The solvent and solution phases coexist inside the cuplike region.

We learn from experiment that the case $N=10$ is in the single phase region at room temperature $[33,35]$ and therefore has low $\bar{\chi}$. We can thus mark the concentration $f_{10}=f_{\text {exp }}$. At the same experimental concentration with $N=9$ we end up in the biphase region and phase separate to a pure solvent and solution with concentration $f_{9}>f_{\text {exp }}$. The condition $N=8$ will give even higher $\bar{\chi}$ and hence even lower $f_{8}$ in the solution domains. The conclusion is that the "local" concentration of membranes will increase as $N$ is decreased. Since $p$ (meeting) $\sim f^{2}$, the probability for cross-links $p$ (cross-link) increases even faster with reducing $N$. We also assume that $p$ (ordered) either decreases with increasing $N$ or its maximum is not a pronounced one but is masked by the effect described above.

\section{EXPERIMENT}

\section{A. Materials}

Figure 2 shows the chemical structures of the PFs used in this work. The molecular characteristics of these polymers are compiled in Table I. PF6-PF10 were prepared following the Yamamoto-type polycondensation of the corresponding 


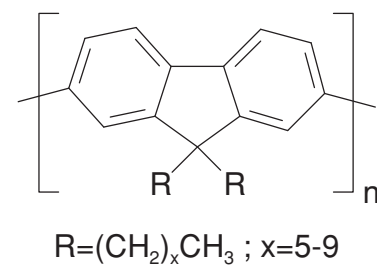

FIG. 2. Chemical structure of PFs with linear side chain $R$. The side chain length $N$ varied from 6 (PF6) to 10 (PF10) carbons $(N=$ $x+1)$.

2,7-dibromo-9,9-dialkylfluorenes with $\mathrm{Ni}(\mathrm{COD})_{2}$ [8]. The molecular weights were determined using gel permeation chromatography. The polymer overlap concentrations were calculated using Eqs. (1) and (2), where $M_{n} / N_{A}$ was used as the weight of a single molecule. The values of the parameters used for the repeat length and persistence length for linear side chain PFs were the repeat length of PF8 [39] ( 8.3 $\AA)$ and the average of the persistence length found for PF8 in toluene in Refs. [19] $(85.5 \pm 10.5 \AA)$ and [29] ( $~ 98 \AA)$. The total lengths of the polymers estimated from the degree of polymerization and the repeat length of PF8 fall within the limits 1000-2000 ̊.

For neutron scattering, the PFs were dissolved in deuterated MCH (MCH- $\left.d_{14}\right)(99.5 \%$ D, Apollo Scientific Ltd.) and for x-ray scattering in $\mathrm{MCH}$ (Sigma-Aldrich). The employed concentration $c$ was $10 \mathrm{mg} / \mathrm{mL}$ corresponding to $1.29 \mathrm{wt} \%$. The volume fractions are shown in Table I. Only PF10 is soluble in $\mathrm{MCH}$ or $\mathrm{MCH}-d_{14}$ at room temperature at the applied concentration. To achieve a complete solution at elevated temperatures and subsequent gelation [26,32] at low temperatures, the mixtures were prepared via a heating-cooling cycle. In a typical procedure the mixtures were first heated up to $80-85{ }^{\circ} \mathrm{C}$ and stirred at $1000 \mathrm{rpm}$ for $1 \mathrm{~h}$ until completely clear solutions were observed. These samples were then cooled from $80-85{ }^{\circ} \mathrm{C}$ down to $-25{ }^{\circ} \mathrm{C}$ for $1 \mathrm{~h}$ and subsequently warmed to $20^{\circ} \mathrm{C}$ before measurements. After this procedure PF7-MCH- $d_{14}, \mathrm{PF} 8-\mathrm{MCH}-d_{14}$, and PF9-MCH- $d_{14}$ mixtures become turbid and viscous or gel-like at $20{ }^{\circ} \mathrm{C}$, while PF10-MCH- $d_{14}$ appears as a transparent liquid. PF6 is an exception, and due to the poorer solubility, the mixture was typically heated up to $100^{\circ} \mathrm{C}$, a temperature limited by the boiling point of $\mathrm{MCH}\left(101{ }^{\circ} \mathrm{C}\right)$. However, PF6-MCH- $d_{14}$ is not transparent even at $100{ }^{\circ} \mathrm{C}$ and the samples appear macrophase separated.

TABLE I. Molecular characteristics of the PFs used in this work. $M_{n}$ is the number-averaged molecular weight. $M_{w}=$ $\sum_{i} W_{i} M_{i} / \sum_{i} W_{i}$, where $W_{i}$ is the total weight of the $i$ th polymer species. $c^{*}$ and $c$ are the polymer overlap concentration and the concentration of the $\mathrm{MCH}$ or $\mathrm{MCH}-d_{14}$ mixtures.

\begin{tabular}{lcccc}
\hline \hline Material & $M_{n}(\mathrm{~kg} / \mathrm{mol})$ & $M_{w}(\mathrm{~kg} / \mathrm{mol})$ & $c^{*}(\mathrm{vol} \%)$ & $c(\mathrm{vol} \%)$ \\
\hline PF6 & 84 & 200 & $0.104 \pm 0.003$ & 0.97 \\
PF7 & 63 & 144 & $0.138 \pm 0.004$ & 0.99 \\
PF8 & 47 & 153 & $0.181 \pm 0.005$ & 1.00 \\
PF9 & 109 & 221 & $0.134 \pm 0.004$ & 1.01 \\
PF10 & 86 & 236 & $0.149 \pm 0.005$ & 1.03 \\
\hline \hline
\end{tabular}

\section{B. SANS}

SANS measurements were performed at the instrument D11 at the Institut Laue-Langevin (ILL), Grenoble. Scattering intensities were recorded with a two-dimensional positionsensitive ${ }^{3} \mathrm{He}$ detector with an array of $128 \times 128$ cells of $7.5 \mathrm{~mm} \times 7.5 \mathrm{~mm}$ size. To cover a large $q$ range, two wavelengths were used. Instrument settings were as follows: a wavelength of $6 \AA$ with sample-to-detector distances of $1.5,8$, and $34 \mathrm{~m}$, and a wavelength of $16.5 \AA$ with a sample-to-detector distance of $34 \mathrm{~m}$. These settings provide a $q$ range of $0.00058-0.44 \AA^{-1}$. All samples and background solvents were confined in rectangular 2-mm-thick Hellma cells of type $404-\mathrm{QS}$. The cells were placed in a high precision temperature-controlled copper rack kept at $10{ }^{\circ} \mathrm{C}$.

The two-dimensional scattering data obtained were radially averaged and background corrected using the ILL standard data reduction routines. $\mathrm{H}_{2} \mathrm{O}$ was used as secondary calibration standard (calibrated against a monodisperse polymer standard). Data were put on an absolute scale by using the known wavelength-dependent effective cross section of $\mathrm{H}_{2} \mathrm{O}$, determined for D11 with its current ${ }^{3} \mathrm{He}$ detector to $d \Sigma / d \Omega=0.983 \mathrm{~cm}^{-1}$ at $6 \AA$ and $1.843 \mathrm{~cm}^{-1}$ at $16.5 \AA$. The incoherent scattering from polymer hydrogen was taken into account by using a 1:99 mixture of MCH:MCH- $d_{14}$ as a background. Multiple scattering was expected to be negligible due to the high transmission (>85\% at $16.5 \AA$ ).

The initial consideration of SANS data was made using simple scaling arguments. In this consideration the scattering intensity $I(q)$ follows the power law $I(q) \propto q^{-\alpha}$, where the exponent $\alpha=1$ refers to separated rodlike particles and $\alpha=2$ to sheetlike particles. When the data pointed to rodlike particles, they were fitted by means of indirect Fourier transform (IFT) and a model of cylindrical particles with the software GNOM [40]. This consideration was enhanced by a two-component model fitted to the entire scattering curve. The low $q$ part of the curve was fitted to the Debye-Bueche (DB) equation [2]

$$
I(q) \sim \frac{1}{\left(1+\xi_{s}^{2} q^{2}\right)^{2}} .
$$

This equation describes an interconnected two-phase system and $\xi_{s}$ is related to the typical size of these phases. When the sample was expected to contain rigid particles, the high $q$ region was fitted to an Ornstein-Zernike- (OZ) type equation [27]

$$
I(q) \sim \frac{1}{1+\xi_{d} q \exp \left(q^{2} R^{2} / 4\right)},
$$

where $\xi_{d}$ represents the mesh size of the dynamic network and $R$ is the radius of the rodlike particle, ultimately the radius of a single rodlike polymer. When the sample was expected to contain sheetlike particles, the high $q$ part was fitted to the equation [33]

$$
I(q) \sim \frac{1}{1+\xi_{\text {sheet }}^{2} q^{2} \exp \left(q^{2} L^{2} / 12\right)},
$$

where $\xi_{\text {sheet }}$ represents the lateral size and $L$ is the thickness of a polymer sheet. The models are described in further detail in Refs. [2,27,33]. 


\section{SAXS and WAXS}

SAXS and WAXS measurements of PF7-PF10 in MCH were carried out at beam line B1 on the DORIS III synchrotron at HASYLAB, Hamburg [41]. A photon energy of $16 \mathrm{keV}$ was selected to cover the $q$ range $0.006-4.29 \AA^{-1}$ by measuring SAXS at sample-to-detector distances of 88.4 and $358.4 \mathrm{~cm}$. The SAXS patterns were measured using a single photon counting two-dimensional pixel detector, Pilatus 300k (Dectris). WAXS patterns were measured simultaneously using a one-dimensional strip detector, Mythen (Dectris), which was placed at a $13.3 \mathrm{~cm}$ distance from the sample, allowing for overlap between SAXS and WAXS data. The beam size on the sample was $0.7 \mathrm{~mm} \times 0.7 \mathrm{~mm}$. The $\mathrm{MCH}$ background was subtracted from the data before uniting measurements made at different distances. The total measurement time per sample was about $3.5 \mathrm{~h}$. The samples were in quartz capillaries (diameter $1.5 \mathrm{~mm}$ ). Multiple scattering was expected to be negligible due to the high transmission ( 90\%).

WAXS measurements of PF6-MCH samples were carried out at room temperature using a rotating anode setup powered with a Rigaku UltraX18S generator $(18 \mathrm{~kW})$. The $\mathrm{x}$ rays were focused and monochromatized with bent $\mathrm{Cu}$ /glass mirror and bent asymmetric $\mathrm{Si}(111)$ crystal providing a spectrally pure $\mathrm{Cu}$ $K \alpha_{1}(\lambda=1.5405 \AA)$ beam $(0.3 \mathrm{~mm} \times 1.2 \mathrm{~mm}$ on the detector $)$. The data were collected using a Mar345 image plate detector $150 \mathrm{~mm}$ from the sample position. The sample was sealed in a Hilgenberg glass capillary with $0.01 \mathrm{~mm}$ wall thickness and $2 \mathrm{~mm}$ diameter.

\section{Optical spectroscopy}

The optical absorption spectra were measured using a Shimadzu UV-3600 spectrophotometer over 320-500 nm at $0.5 \mathrm{~nm}$ step size. The sample cells comprised two microscope slides that were cleaned in nitric acid, water, and acetone. For all polymers, a drop of hot isotropic solution $\left(\sim 90{ }^{\circ} \mathrm{C}\right)$ was added onto one slide while the other was pressed on top and the edges were clipped together. The samples were left at room temperature for $10 \mathrm{~min}$ and initial absorption spectra were measured. The samples were subsequently placed in a dewar filled with dry ice for $45 \mathrm{~min}$. The outer surfaces of the slides were then cleaned using compressed air to remove ice that forms when the samples are removed from the dry ice. The absorption spectra were measured after this procedure at room temperature. The absorption spectrum of the blank sample cell was subtracted as a background. Data were fitted to the spectral peaks corresponding to free polymer and polymer assemblies with and without $\beta$ phase.

\section{RESULTS AND DISCUSSION}

Figure 3 plots SANS patterns of PF8-MCH- $d_{14}$ normalized to $c$ for $c=1-10 \mathrm{mg} / \mathrm{mL}$ at $10{ }^{\circ} \mathrm{C}$. As expected, $10 \mathrm{mg} / \mathrm{mL}$ PF8-MCH- $d_{14}$ shows a distinctive power-law exponent $\alpha \sim 2$ over the $q$ range $>0.004 \AA^{-1}$, which has been interpreted as sheetlike polymer assemblies in Ref. [26]. These sheets can be denoted as membranes, when they appear as bilayers [33]. Figure 3 shows that the $10 \mathrm{mg} / \mathrm{mL}$ mixture follows an exceptionally uniform power law with the exponent $2.26 \pm 0.01$ also beyond the previously reported $q$ range, altogether over

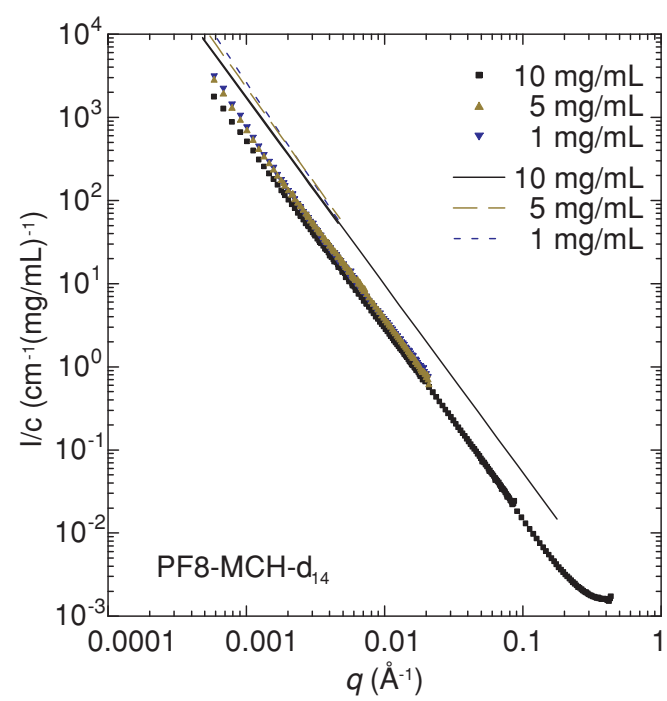

FIG. 3. (Color online) SANS patterns of PF8-MCH- $d_{14}$ with concentrations of 10 (black squares), 5 (dark yellow up triangles), and $1 \mathrm{mg} / \mathrm{mL}$ (blue down triangles). Also shown are the best linear fits for comparison (offset for clarity). $T=10^{\circ} \mathrm{C}$.

more than two orders of magnitude (0.00058-0.1 $\left.\AA^{-1}\right)$. This indicates that the sheets can be larger $(>3000 \AA)$ than previously expected $(\sim 1000 \AA)$ [26]. The nearly constant $\alpha$ over large $q$ range together with the fact that $1<\alpha<3$ but not exactly 2 , can also indicate a structure which can be understood as a mass fractal with the dimension $D_{m} \approx 2.3$ (see Ref. [42] for details). This behavior is maintained for lower concentrations except with a slight deviation at the lowest $q$. The best linear fits for $q=0.00058-0.004 \AA^{-1}$ for 1,5 , and $10 \mathrm{mg} / \mathrm{mL}$ mixtures correspond to the slopes $-2.49 \pm 0.02$, $-2.33 \pm 0.02$, and $-2.27 \pm 0.01$, respectively. This means that the system is similar regardless of the studied concentration. However, it may not be understood as independent scatterers of separated particles but rather as a continuous system of polymer sheets whose overall shape does not change with increasing distances between the sheets.

Figure 4 plots SANS patterns of $10 \mathrm{mg} / \mathrm{mL} \mathrm{PF}_{N}-\mathrm{MCH}-d_{14}$ with increasing $N$ at $10{ }^{\circ} \mathrm{C}$. The best linear fits (offset for clarity) illustrate linear sections of the data. Also shown are fits to the IFT and DB-OZ models for selected data. Structural parameters estimated from the fits are shown in Table II. PF6-PF9 $\mathrm{MCH}-d_{14}$ show a predominantly -2 slope and PF10-MCH- $d_{14}$ shows a -1 slope in the $q$ range $>0.004 \AA^{-1}$, which has been studied previously. This kind of change in the slope for an individual polymer would mean a phase transition from sheets to separated rodlike polymers (denoted as the membrane-isotropic transition in Ref. [33]). The transition temperature $T_{\mathrm{mem}}^{*}$ decreases with increasing $N$ and for PF10 it is lower $\left(-5^{\circ} \mathrm{C}\right)$ than the measurement temperature, which explains its -1 decay in Fig. 4.

The deviations from the ideal -2 and -1 slopes are also significant and are interpreted to originate from the limited lateral size $\left(<0.004 \AA^{-1}\right)$ and the thickness and internal structure $\left(\sim 0.1 \AA^{-1}\right)$ of the sheetlike and rodlike particles [32]. The areas of these deviations are marked by arrows a and $b$ in Fig. 4. As detailed in Ref. [32], the dimensions of the sheets 


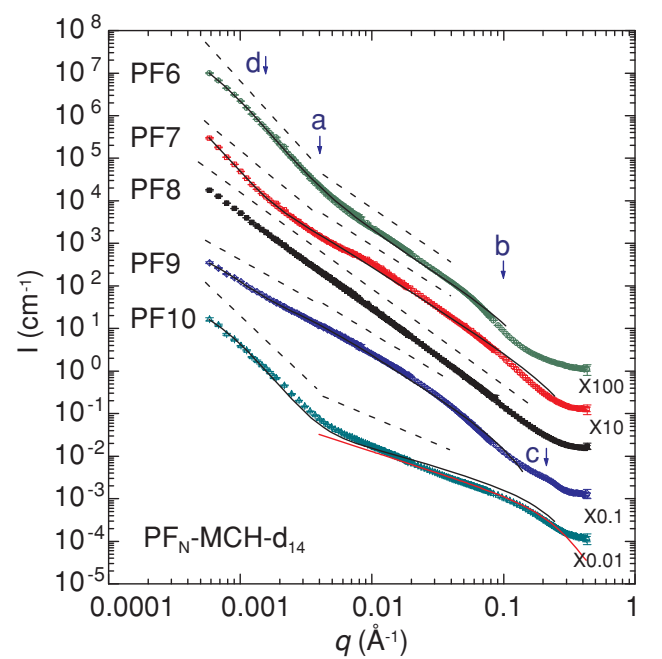

FIG. 4. (Color online) SANS patterns of PF6 (green open spheres), PF7 (red open squares), PF8 (black solid squares), PF9 (blue open diamonds), and PF10 (cyan open up triangles) mixed with $\mathrm{MCH}-d_{14}$. Concentration was $10 \mathrm{mg} / \mathrm{mL}$ in all cases. The curves are offset for clarity, with PF8 unscaled. Black solid lines are fits to Eqs. (9)-(11). A red solid line is a fit to the IFT model for PF10. Arrows mark approximate $q$ regions connected with the lateral size, a, and thickness of the sheets, b, an interference maximum, c, and the network of sheets, d. Dotted lines show linear fits (offset for clarity). $T=10^{\circ} \mathrm{C}$.

vary in an "odd-even" fashion. The sheets appear laterally larger for PF6 and PF8 and smaller for PF7 and PF9, which is reflected by a shallow shoulderlike deviation from the nearly -2 slope (arrow a) for PF7 and PF9 but not for PF6 and PF8. Sheets are also thinner for PF8 than for PF7 or PF9 as indicated by the deviation from the -2 slope at higher $q$ seen for PF7 and PF9 but not for PF8. Thicker sheets produce an interference maximum at $\sim 0.25 \AA^{-1}$ (arrow c) that for contrast reasons [32], is better resolved in X-ray scattering data (vide infra).

Attention should be placed on the low $q$ part of the data $\left(q<0.004 \AA^{-1}\right)$ that have not been reported to date. None of the curves level off distinctively, but instead show a slight downturn of the slope in PF9-MCH- $d_{14}$, a continuous increasing tendency in PF8-MCH- $d_{14}$, or an upturn in PF6 and $\mathrm{PF} 7-\mathrm{MCH}-d_{14}$. These all point to the larger length scale agglomerates or density fluctuations at lower $N$ in the samples containing polymer sheets. The exponent $\alpha$ decreases with increasing $N$ (Table II). This implies that the larger length scale agglomerates are more compacted for smaller $N$. This phenomenon may be understood in terms of cross-link probability as outlined in Sec. II. Elsewhere, similar slope dependence has been reported for inhomogeneous polyacrylamide gels and is also interpreted in terms of cross-link density [43].

Moreover, comparing PF6 to PF7, the exponent shows a crossover from $\alpha$ in the range 3-4 to $\alpha$ being in the range $1-3$ in the $q$ range $0.00058-0.004 \AA^{-1}$, which could indicate a surface fractal structure for PF6 and a mass fractal structure for PF7-PF9. In this interpretation PF6 would form separated particles containing some $\mathrm{MCH}$, the scattering dominated by the particle surface of randomly distributed sheets. PF7-PF9 would exist as very large sheets with a varying amount of holes, so essentially as networks of smaller sheets. PF7 represents a borderline case and shows a mass fractal-type slope for $q=$ $0.00058-0.002 \AA^{-1}$.

The data of PF10 do not level off at low $q$ but also show an upturn and density fluctuations with the "characteristic distance" $\xi_{s} \sim 1700 \AA$. We expect that PF10 behaves similarly to PF8 in high concentrations in toluene [27]. As $c>c^{*}, \mathrm{PF} 10$ chains are overlapping and density fluctuations can well exist without any macroscopic accumulation.

Chen et al. [30] studied initially isotropic PF8-MCH over a broad concentration range $(0.001-3$ wt $\%$ or $\sim 0.0008-$ $2.3 \mathrm{vol} \%)$ at room temperature by aging it over several

TABLE II. Parameters estimated from the fits to the SANS data of $10 \mathrm{mg} / \mathrm{mL} \mathrm{PF}{ }_{N}-\mathrm{MCH}-d_{14} . \alpha$ stands for the power-law exponent for a given $q$ range. $\xi_{s}$ is the correlation distance in the interconnected two-phase system. $\xi_{\text {sheet }}$ and $L$ are the size and thickness of the polymer sheets. $\xi_{d}$ and $R$ are the mesh size and radius of rodlike objects. $R_{\mathrm{CS}, g}$ and $D_{\max }$ represent the cross-sectional radius of gyration and the maximum diameter of the cylindrical scatterer.

\begin{tabular}{|c|c|c|c|c|c|c|}
\hline \multirow[b]{2}{*}{ Model } & \multicolumn{6}{|c|}{ Polymer } \\
\hline & & $\begin{array}{c}\text { PF6 } \\
\text { Sheet }\end{array}$ & $\begin{array}{c}\text { PF7 } \\
\text { Sheet }\end{array}$ & $\begin{array}{c}\text { PF8 } \\
\text { Sheet }\end{array}$ & $\begin{array}{l}\text { PF9 } \\
\text { Sheet }\end{array}$ & $\begin{array}{c}\text { PF10 } \\
\text { Rod }\end{array}$ \\
\hline \multicolumn{7}{|c|}{ Analysed q range $\left(\AA^{-1}\right)$} \\
\hline \multirow[t]{4}{*}{$\alpha$} & $0.00058-0.004$ & $3.40 \pm 0.02$ & $2.55 \pm 0.04$ & $2.27 \pm 0.01$ & $1.74 \pm 0.01$ & $2.96 \pm 0.02$ \\
\hline & $0.00058-0.002$ & & $3.03 \pm 0.06$ & & & \\
\hline & $0.004-0.04$ & $2.10 \pm 0.01$ & $2.23+0.01$ & & $1.87 \pm 0.01$ & $1.29 \pm 0.01$ \\
\hline & $0.00058-0.1$ & & & $2.26 \pm 0.01$ & & \\
\hline$\xi_{s}(\AA)$ & $0.00058-0.1$ & $2016 \pm 12$ & $>3530$ & & $>1500$ & $1670 \pm 20$ \\
\hline$\xi_{\text {sheet }}(\AA)$ & & $>450$ & $476 \pm 13$ & & $428 \pm 8$ & \\
\hline$L(\AA)$ & & $22 \pm 15$ & $11 \pm 6$ & & $25 \pm 4$ & \\
\hline$\xi_{d}(\AA)$ & & & & & & $>320$ \\
\hline$R(\AA)$ & & & & & & $\sim 8$ \\
\hline$R_{\mathrm{CS}, g}(\AA)$ & $0.004-0.43$ & & & & & $5.2 \pm 0.1$ \\
\hline$D_{\max }(\AA)$ & & & & & & $\sim 15$ \\
\hline
\end{tabular}



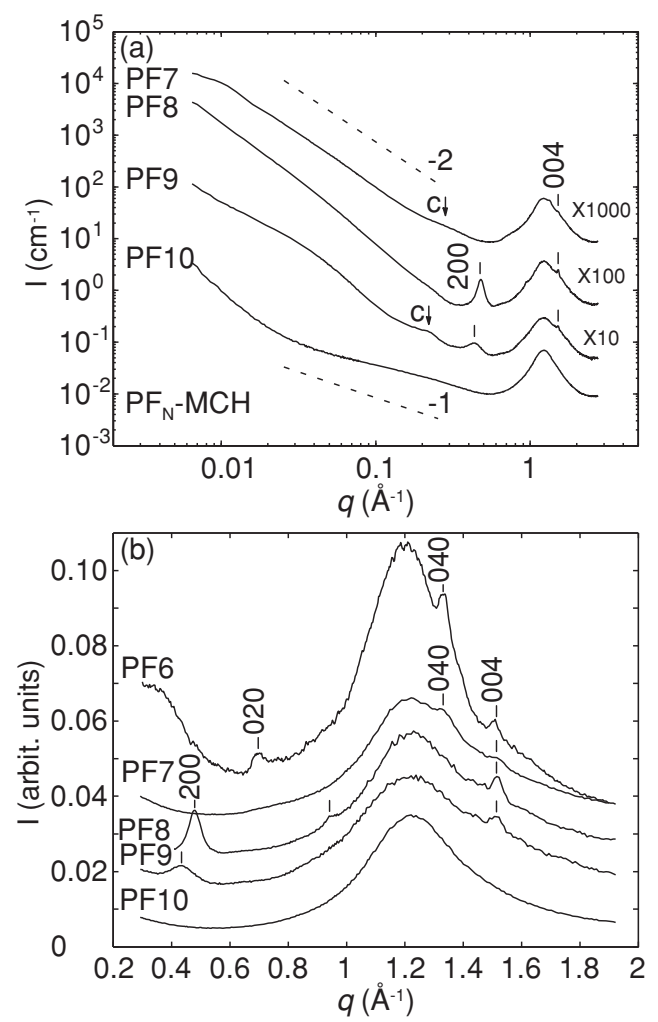

FIG. 5. (a) SAXS and WAXS patterns of $\mathrm{PF}_{N}-\mathrm{MCH}$ for $N=$ 7-10 and (b) WAXS pattens for $N=6-10$. An interference maximum, c, corresponds to that shown in Fig. 4. Curves are offset for clarity. $c=10 \mathrm{mg} / \mathrm{mL}$ for $N=7-10$ but $20 \mathrm{mg} / \mathrm{mL}$ for $N=6$. $T \sim 23{ }^{\circ} \mathrm{C}$.

days. The authors found similar gelation phenomenon as described earlier [32,33] and in this paper arising from a more rapid heating-cooling cycle. They put forward an idea of interconnected PF8 morphology and the idea of concentration fluctuations amongst the sheets. Chen et al. found, in particular, that the gelation is driven by a macrophase separation that leads to the coexistence of sheets (what they called the liquid crystalline phase) and isotropic liquid [30]. True macrophase separation eventually takes place. We find that this macrophase separation process is facilitated by decreasing $N$, such that PF6 is actually always macrophase separated and does not form an isotropic $\mathrm{MCH}$ solution even with heating. PF10 forms a gel upon cooling but stays transparent at room temperature in MCH without signs of macrophase separation.

Figure 5(a) shows the SAXS and WAXS curves of $\mathrm{PF}_{N^{-}}$ $\mathrm{MCH}$. The slopes seen in the SAXS data are consistent with the SANS data. The broad maximum at $1.2 \AA^{-1}$ originates from the solvent $\mathrm{MCH}$ as its dominance does not allow perfect background subtraction. An interference maximum, c, which is visible in SANS data, is resolved at least for PF9 and may correspond to a very broad feature seen for PF7, also marked by c. This may indicate a two-layer periodic membrane or a single layer of $40 \AA$ thickness.

Figure 5(b) plots the WAXS curves on a linear scale. The data show the existence of relatively well-ordered, crystallinelike regions for the samples PF6-PF9. The observed reflection patterns have similarities to the $\beta$ phase found in as-cast or solvent-exposed films of PF8 [39]. PF10 does not show any reflections as expected for the sample dominated by locally free chains.

The reflections can be indexed along three crystallographic axes. The order along the $\mathbf{c}$ axis is characterized by a sharp reflection at $1.51 \AA^{-1}$ which is observed for all the gel-like samples and corresponds to half of the monomer repeat $(4.17 \AA)$. As the identity period in the solid state $\beta$ phase is two monomers [39], we denote it as 004. The solid state $\beta$ phase involves stacking of relatively well-ordered layers with $12-15 \AA$ periodicity [17]. This order along the a axis is characterized by a reflection, 200 , at $0.4-0.5 \AA^{-1}$ and its possible multiples. In the present samples only PF8 and PF9 show a clear 200 reflection and supposedly, domains whose structure corresponds to the solid state $\beta$ phase. The calculated thicknesses of the ordered domains are about $120 \AA$ for PF8 and $70 \AA$ for PF9 which are far below those reported for crystalline solid state phases [15] but are somewhat larger than the estimated thicknesses for membranes (Table II). A sharp reflection at 1.33-1.34 $\AA^{-1}$ is observed for PF6 and PF7 and another one at $0.69 \AA^{-1}$ for PF6 corresponding to the distance of $4.7 \AA$. We suggest that these indicate order along the $\mathbf{b}$ axis and index them as 040 and 020 in accordance with our earlier work [32].

Thus, although the solid state $\beta$ phase shows threedimensional ordering, the present gel samples only show two-dimensional ordering. PF6 and PF7 show order in the $(0 k l)$ plane and PF8 and PF9 in the $(h 0 l)$ plane. The latter structure is deemed to be the $\beta$ phase. The ordered domains are more rigid for PF6 and PF7 and less rigid for PF8 and PF9, allowing more lateral motion for the chains within the sheets. This difference may explain differences in the way the ribbons are constructed, if we just simply assume that the observed ordered two-dimensional structures are part of the larger length scale architecture observed in small-angle scattering experiments.

Figure 6 shows the optical absorption spectra of $\mathrm{PF}_{N}-\mathrm{MCH}$ at $10 \mathrm{mg} / \mathrm{mL}$ with the modeled fits to the spectra including the 0-0 peak, three vibronic peaks with several multiplicities, as well as a distinctive asymmetric peak at $2.9 \mathrm{eV}$. The data are qualitatively similar to those reported earlier [32,34] but the modeling allows us to estimate the relative fractions of polymer assemblies and locally free chains. The vibronic replicas used correspond to modes with energies of $85 \mathrm{meV}(v 1), 156 \mathrm{meV}$ (v2), and $199 \mathrm{meV}$ (v3) which were selected from fits to the $\beta$-phase PF8 photoluminescence spectrum and which agree well with Raman spectroscopy of PF8 [44]. PF6 and PF7 show scattering effects from macrophase separation around $2.7 \mathrm{eV}$. The spectral shape constrains the fits well over the range 2.8-3.4 eV but the fits to the remainder of the spectrum above $3.4 \mathrm{eV}$ are subject to uncertainty. However, we are focusing only on information derived from the fits to the range $2.8-$ $3.4 \mathrm{eV}$.

Figure 7 shows the Huang-Rhys factors of the main spectral components and the area of asymmetric peak as a function of $N$. The interfluorene stretch mode $v 2$ increases at the expense of the phenyl breathing stretch mode $v 3$. The first $v 1$ peak is attributed to the locally free chains without intermolecular contacts, while $v 2$ and $v 3$ are associated with the loose membranes and ordered sheets. The $v 2$ peak increases at the expense of the $v 3$ peak with increasing $N$, 

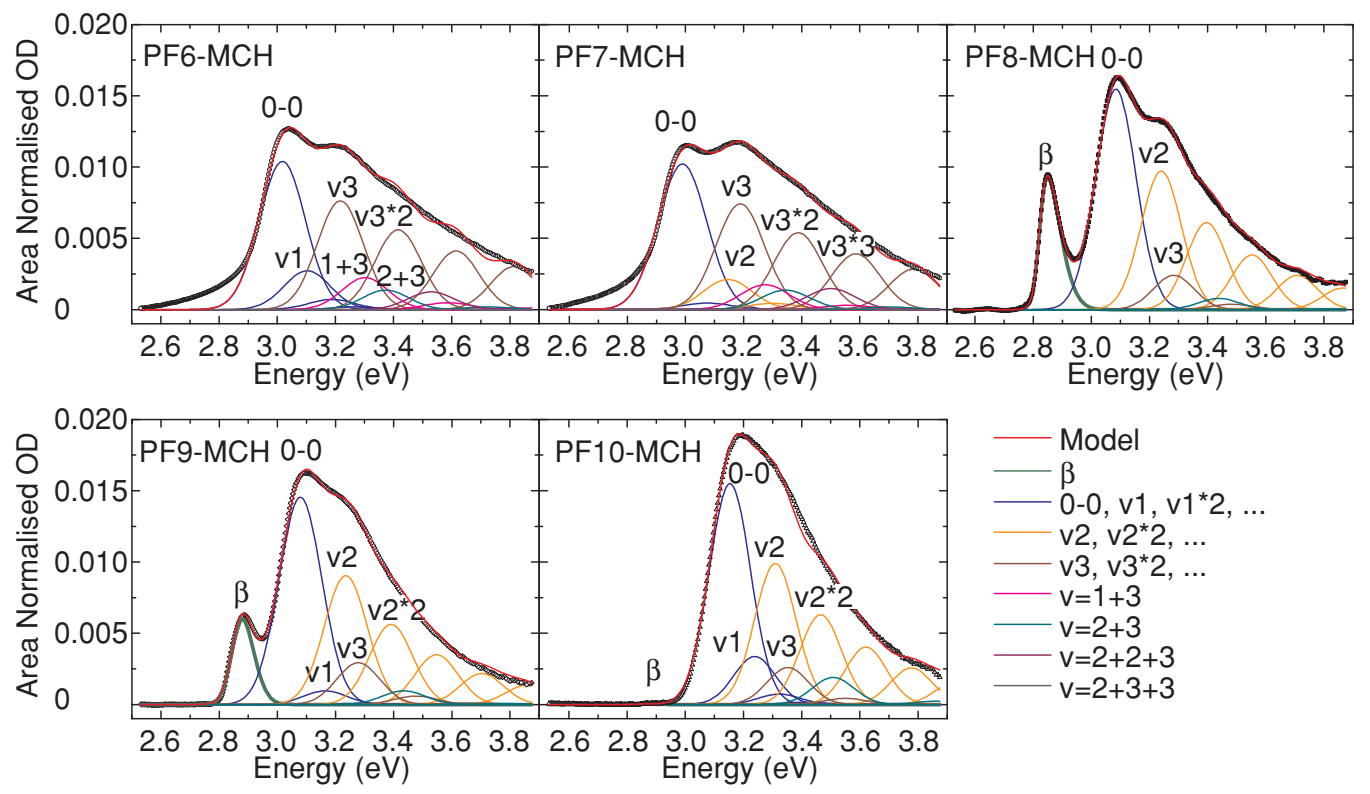

FIG. 6. (Color online) Area normalized optical density (OD) of $\mathrm{PF}_{N}-\mathrm{MCH}$ for $N=6-10$ (black symbols) and the overall model fitted to the data (red solid line). Also shown are fits to the spectral components $0-0$ and $v 1$ (blue), $v 2$ (orange), $v 3$ (brown), $v=(1+3)(\mathrm{magenta}), v=$ $(2+3)$ (cyan), $v=(2+2+3)$ (purple), and $v=(2+3+3)$ (dark gray solid line) and their multiplicities as well as the fit to the $\beta$ peak (green). The notation $v n^{*} m$ refers to the $m$ th replica of the vibrational mode $n . T \sim 20{ }^{\circ} \mathrm{C}$.

thus pointing to the increasing fraction of membranes and decreasing fraction of ordered sheets. The intrinsic stiffness of polymer backbones is similar for all $N$, as indicated by similar room temperature spectra before annealing for dilute solutions [34]. The asymmetric peak at $2.85 \mathrm{eV}$ is indicative of the amount of $\beta$ phase present. This varies in a nonlinear fashion and finds its maximum at $N=8$. These results are consistent with the WAXS data, thus indicating the existence of the $\beta$ phase for $N=8-9$, restricted motion along the long chain axis for $N=6-7$, and larger freedom to vibrate for $N=8-9$.

Figures 8 and 9 summarize the structural ideas deduced from the theory and experiment. Chen et al. [30] studied PF8-MCH and concluded that this system contains areas

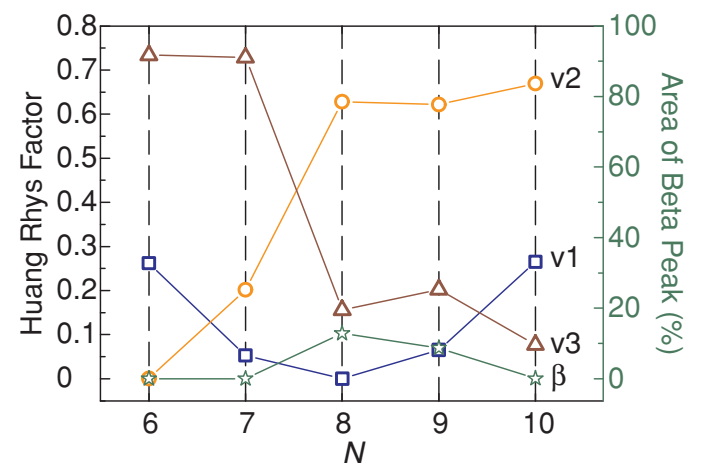

FIG. 7. (Color online) Huang Rhys factors of vibrational components $v 1^{*} 1$ (blue open squares), $v 2^{*} 1$ (orange open circles), and $v 3^{*} 1$ (brown open up triangles) and the area of $\beta$ peak (green open stars) of $\mathrm{PF}_{N}-\mathrm{MCH}$ for $N=6-10$. These values are estimated from the optical absorption data shown in Fig. 6. The dotted lines correspond to the experimentally studied $N$. of "liquid crystal" phase with spatial fluctuations coexisting with an isotropic phase (cf. Fig. 8). We suggest that at room temperature the same holds for PF6-MCH, PF7-MCH, and $\mathrm{PF9}-\mathrm{MCH}$ that contain polymer sheets that are not isotropically distributed but form "liquid crystalline" areas within the sample. The liquid crystals (in our notation sheets or membranes) are metastable and the system becomes macrophase separated over time. The tendency to macrophase separate with time increases with decreasing $N$ so that PF6$\mathrm{MCH}$ appears largely macrophase separated. This tendency follows with increasing $T_{\mathrm{mem}}^{*}$. The polymers PF7-PF9, though in our experiment deep in the membrane phase region, show

(a) segregated networks of polymer sheets

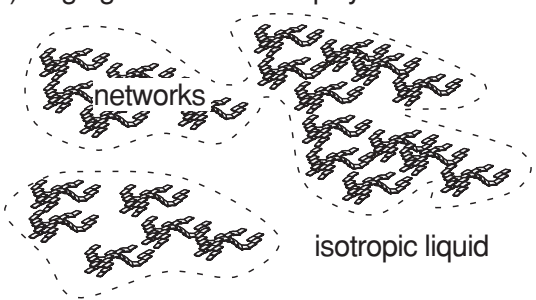

(b) isotropic phase of overlapping chains

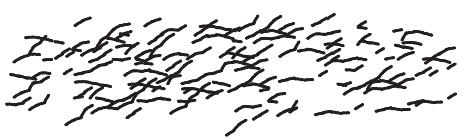

FIG. 8. Illustration of macroscopic appearances of $\mathrm{PF}_{N}-\mathrm{MCH}$ at room temperature for $c \sim 1$ vol \%. (a) Isotropic liquid areas and areas of polymer agglomerates, denoted as liquid crystalline phase in Ref. [30]. This is denoted as a lyotropic phase with solvent coexistence in Ref. [33] and corresponds to PF6-PF9-MCH. (b) Macroscopically isotropic phase of overlapping but locally free chains. This corresponds to PF10-MCH. 
(a)

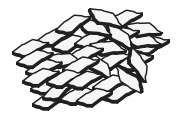

PF6-MCH

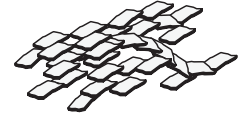

PF7-MCH

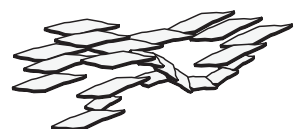

$\mathrm{PF} 8-\mathrm{MCH}$

$T_{\text {mem }}{ }^{*}<T$

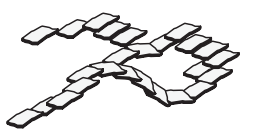

PF9-MCH

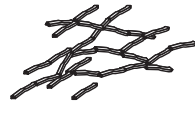

PF10-MCH

$T_{\text {mem }}{ }^{*}>T$

increasingly open superstructure / decreasing tendency to macrophase separation

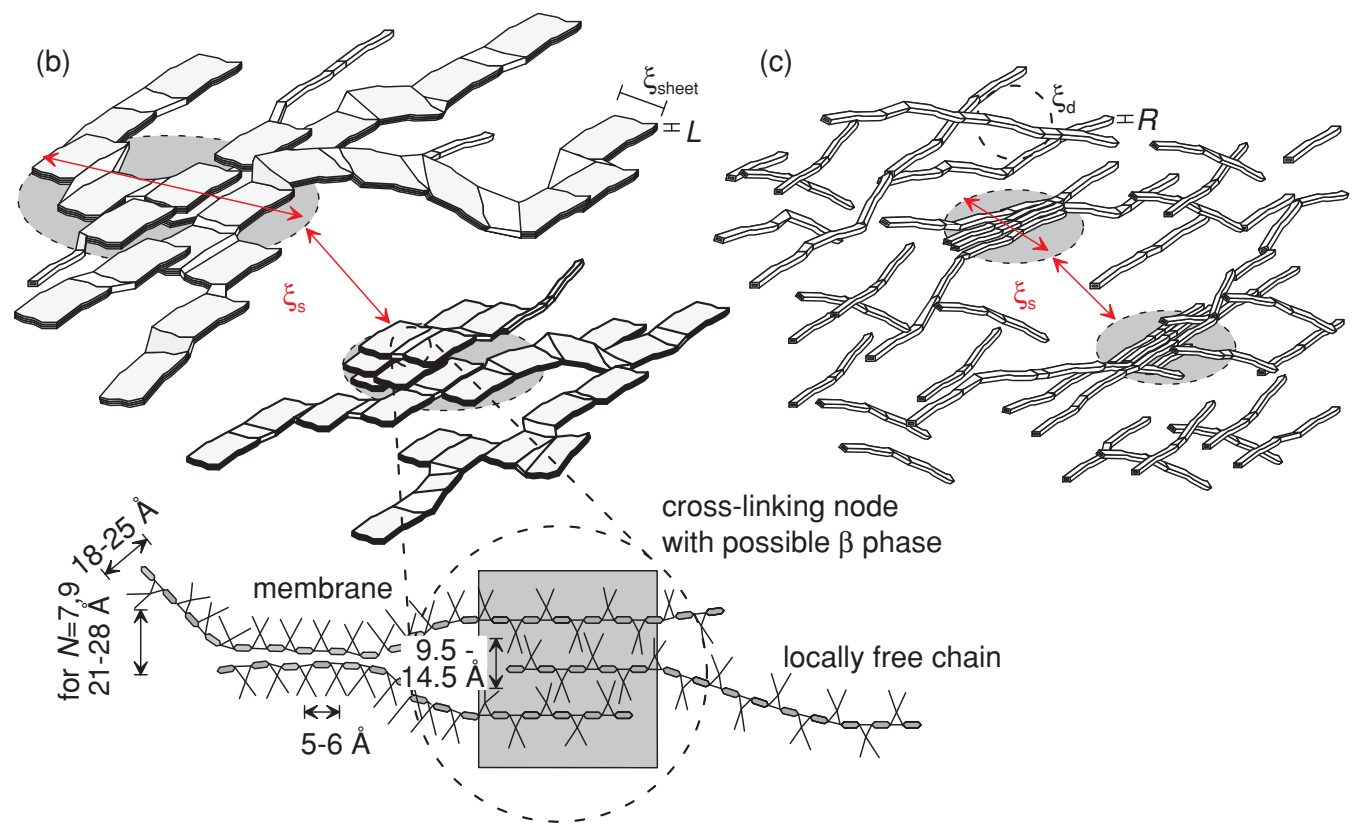

FIG. 9. (Color online) (a) An overview of increasingly open ribbonlike agglomerates of $\mathrm{PF}_{N}$ assemblies in $\mathrm{MCH}$ with increasing $N . T$ corresponds to the fixed experimental (room) temperature while $N^{*}$ and $T_{\mathrm{mem}}^{*}$ refer to the nematic-membrane and membrane-isotropic phase boundaries as defined in Ref. [33]. (b) Agglomerates of sheetlike assemblies and (c) overlapping locally separated rodlike polymers. Also shown are parameters that characterize the size of sheets $\left(\xi_{\text {sheet }}, L\right)$ or rodlike polymers $\left(\xi_{d}, R\right)$. Red arrows (see the online version) indicate density fluctuations potentially associated with the parameter $\xi_{s}$. The ribbons (b) contain loose membranes, weakly ordered areas, and to some extent locally free chains.

a well-defined $T_{\mathrm{mem}}^{*}$, while PF6 does not reach an isotropic phase at any experimentally attainable temperature [33].

The thickness $(L)$ and lateral size of sheets $\left(\xi_{\text {sheet }}\right)$ are nearly independent of $N$ corresponding to the bilayers with aspect ratios of 10-20 (Table II). The sheets are expected to grow until they bend or meet other sheets forming larger ribbonlike agglomerates (Fig. 9). We suggest that the ribbons form an interconnected two-phase system (of ribbons and voids). The arrangements of ribbons seem to become less compact with increasing $N$. PF6 appears most compact with a 3D structure with a disordered surface, whereas PF9 appears as long ribbons forming networks with disordered (mass fractal) interiors.

The connections between ribbons may occur via ordered nodes that contain ordered sheets and/or $\beta$ phase. The approximate size of the ordered domains is well below that of the solid state crystallites. There are two other possible paths for ribbon formation. First, as PF6-MCH and PF7-MCH do not contain $\beta$ phase, the ribbons may simply be packed together forming large compact agglomerates. Second, as the systems with $N \geqslant 8$ contain increasing amounts of locally free individual polymer chains, these chains may act as "tie" molecules connecting separate membranes together.

PF10 appears as locally free polymers that are overlapping and forming a loose network over a larger distance, corresponding to the isotropic phase regime $T>T_{\mathrm{mem}}^{*}$ [33]. PF10-MCH also has density fluctuations but the nodes are not ordered as indicated by nonexistent x-ray diffraction maxima. The nodes may correspond to the segmental alignment reported for PF8 in more dense $(\geqslant 3 \%)$ toluene mixtures [27]. PF10-MCH shows a trace of $\beta$ phase but its fraction is so small $(\ll 1 \%)$ that it cannot play a role in the network formation.

Surin et al. [45] reported ribbons hundreds of nanometers long in solid state PF8 slowly crystallized from toluene. Ribbonlike agglomerates formed by evaporation of the solution are known elsewhere, for example, for poly[9,9-di (2-ethylhexyl)fluorene] [46] or poly[2,5-bis(3-alkylthiophen2-yl)thieno(3,2-b)thiophene] [47,48].

What is generally denoted as $\beta$ phase may refer either to the planar chains [13] or planar chains in the solid films treated by solvent vapor [17] or in solvent mixtures where solvent is the majority component. The question is, to what extent is chain planarization a result of intermolecular interactions that lead to the $\mathrm{PF}_{N}$ aggregation in $\mathrm{MCH}$ (see Sec. II)? Bright et al. [34,35] suggest that chain planarization in $\mathrm{PF}_{N}-\mathrm{MCH}$ is the result of side chain interactions between separate chromophores, even if the two interacting segments are on the same chains.

The extent of intermolecular interactions may be discussed in terms of $c^{*}$, which depends on $R_{g}$, which in turn depends on 
molecular weight and measurement temperature. Chen et al. [29] used PF8 with $M_{n}=128 \mathrm{~kg} / \mathrm{mol}$ and measured $R_{g} \approx 250$ and $460 \AA$ in toluene at $T=25^{\circ} \mathrm{C}$ and $50{ }^{\circ} \mathrm{C}$, respectively, and concluded that the characteristics of planar chains are predominantly present above $c^{*}$ following gelation.

Elsewhere, Kitts and Vanden Bout [24] worked with PF8 $\left(M_{n}=34.8 \mathrm{~kg} / \mathrm{mol}\right.$ ) in $0.001 \mathrm{wt} \%$ (or $\sim 0.0009$ vol \%) toluene and Dias et al. [25] with PF8 $\left(M_{n}=188 \mathrm{~kg} / \mathrm{mol}\right)$ in $3 \mu \mathrm{g} / \mathrm{mL}$ (or $0.0003 \mathrm{vol} \%$ ) $\mathrm{MCH}$ and observed optical characteristics of $\beta$ phase when cooling the solutions (down to $\sim 200 \mathrm{~K}$, for example). Our simple consideration gives values $c^{*} \sim 0.09 \mathrm{vol} \%$ and $\sim 0.05 \mathrm{vol} \%$, implying that the condition $c \ll c^{*}$ holds for both cases. Kitts and Vanden Bout note that "the $\beta$-phase domains" tend to associate at lower concentrations but lead to visual gelation at higher toluene concentrations ( $1 \mathrm{wt} \%$ or 0.9 vol $\%$, i.e., $c>c^{*}$ ) on cooling [24]. Similarly, Bright et al. [35] found the $\beta$-phase domains but no gelation for $c \ll c^{*}$.

These findings give us the following scenarios. First, the optical characteristics of $\beta$ phase could arise from individual polymers for $c \ll c^{*}$. Alternatively, the polymer-polymer interactions could occur within the same polymer chain. This scenario is well justified, if main chains are much longer than the persistence length $L \approx 40 l_{p}$ [25]. If separated in the first place, the polymers may also reassociate, contrary to the classical picture [36].

In light of the present results for $c>c^{*}$, we suggest the scenario where the $\beta$ phase is understood as a sheetlike polymer assembly, but one which can be present regardless of the above-described ribbons. The $\beta$ phase as a polymer aggregation can be observed for $c<c^{*}$, but the ribbon formation and visual gelation requires concentration $c>c^{*}$.

The results described above have analogs to the other $\pi$-conjugated polymers in relatively dense solutions. These systems include, for example, poly(2,5-dinonyl paraphenylene ethynylene), forming sheetlike aggregates at lower and fully dissolved chains at higher temperatures in toluene [49] as well as poly(2,3-diphenyl-5-decyl-1,4-phenylene vinylene) [50] and poly[2-methoxy-5-(2'-ethyl-hexyloxy)-1,4phenylene vinylene] (or MEH-PPV) [51] forming a gel with disclike nodes in toluene but not in a better solvent such as chloroform. A clear analog is also seen to the gels of hairy-rod supramolecules [52] or fluorene-based oligomers forming gels but not precipitates in $\mathrm{MCH}$ [53]. The results may also provide an understanding of polyfluorene blends with varying side chain character [54].

\section{CONCLUSIONS}

We studied how $\mathrm{PF}_{N}-\mathrm{MCH}$ gel with $N=6-10$ behave in terms of polymer sheets at room temperature and rationalized this behavior in terms of cross-linking density and blending theory. For $N=6-9, \sim 1 \mathrm{vol} \% \mathrm{PF}_{N}-\mathrm{MCH}$ contains isotropic liquid phase and polymer assemblies phase (lyotropic phase with solvent coexistence) that contain sheet-like assemblies (loose membranes, ordered sheets, and $\beta$ phase) and locally separated chains. The lateral size of sheets has its maximum at $N=8$. The sheets are suggested to form ribbonlike agglomerates that network with increasing openness and a crossover from surface to mass fractal nature with increasing $N$. For $N=10$, a macroscopically isotropic system of overlapping but locally separated chains is present. The systems with $N=6-9$ contain ordered nodes with a high fraction of ordered sheets $(N=6-7)$ or $\beta$ phase $(N=8-9)$ tying ribbons together, whereas the system with $N=10$ contains nodes connecting individual chains together. The proportions of loose membranes and ordered sheets decrease and the proportion of free chains increases with increasing $N$, while the proportion of $\beta$ phase has its maximum at $N=8$. The polymers within the ordered sheets have restricted motion along the long chain axis for $N=6-7$ but more freedom to vibrate for $N=8-9$. Altogether this paper identifies a complex structural hierarchy for a set of polyfluorene gels. As this set serves as a model system, this work may help in understanding the general behavior of other $\pi$-conjugated polymers and PFs in particular. To study the onset of network formation, future research should include SANS studies of more dilute mixtures.

\section{ACKNOWLEDGMENTS}

M. K. thanks J.-H. Chen of Kun Shan University (Taiwan) and H. D. Burrows of the University of Coimbra (Portugal) for discussions.
[1] S. Panyukov and Y. Rabin, Phys. Rep. 269, 1 (1996).

[2] M. Shibayama, Macromol. Chem. Phys. 199, 1 (1998).

[3] M. J. Winokur, in Handbook of Conducting Polymers, edited by T. A. Skotheim and J. R. Reynolds (CRC Press, Boca Raton, FL, 2007), Chapter 17.

[4] H. Sirringhaus, Adv. Mater. 17, 2411 (2005).

[5] M. J. Banach, R. H. Friend, and H. Sirringhaus, Macromolecules 37, 6079 (2004).

[6] Y. Liu, T. Murao, Y. Nakano, M. Naito, and M. Fujiki, Soft Matter 4, 2396 (2008).

[7] D. Neher, Macromol. Rapid. Commun. 22, 1366 (2001).

[8] U. Scherf and E. J. W. List, Adv. Mater. 14, 477 (2002).
[9] A. Monkman, C. Rothe, S. King, and F. Dias, Adv. Polym. Sci. 212, 187 (2008).

[10] J. Zaumseil and H. Sirringhaus, Chem. Rev. 107, 1296 (2007).

[11] R. Abbel, A. P. H. J. Schenning, and E. W. Meijer, J. Polym. Sci., Part A: Polym. Chem. 47, 4215 (2009).

[12] M. Knaapila and M. J. Winokur, Adv. Polym. Sci. 212, 227 (2008).

[13] W. Chunwaschirasiri, B. Tanto, D. L. Huber, and M. J. Winokur, Phys. Rev. Lett. 94, 107402 (2005).

[14] M. Arif, C. Volz, and S. Guha, Phys. Rev. Lett. 96, 025503 (2006).

[15] S. H. Chen, A. C. Su, C. H. Su, and S. A. Chen, Macromolecules 38, 379 (2005). 
[16] S. H. Chen, C. H. Su, A. C. Su, Y. S. Sun, U. Jeng, and S. A. Chen, J. Appl. Crystallogr. 40, s573 (2007).

[17] S. H. Chen, A. C. Su, and S. A. Chen, J. Phys. Chem. B 109, 10067 (2005).

[18] K. Becker and J. M. Lupton, J. Am. Chem. Soc. 127, 7306 (2005).

[19] M. Grell, D. D. C. Bradley, X. Long, T. Chamberlain, M. Inbasekaran, E. P. Woo, and M. Soliman, Acta Polym. 49, 439 (1998).

[20] M. Ariu, D. G. Lidzey, M. Sims, A. J. Cadby, P. A. Lane, and D. D. C. Bradley, J. Phys.: Condens. Matter 14, 9975 (2002).

[21] M. J. Winokur, J. Slinker, and D. L. Huber, Phys. Rev. B 67, 184106 (2003).

[22] C. Rothe, S. M. King, F. Dias, and A. P. Monkman, Phys. Rev. B 70, 195213 (2004).

[23] S. H. Chen, A. C. Su, C. H. Su, and S. A. Chen, J. Phys. Chem. B 110, 4007 (2006).

[24] C. C. Kitts and D. A. Vanden Bout, Polymer 48, 2322 (2007).

[25] F. B. Dias, J. Morgado, A. L. Maçanita, F. P. da Costa, H. D. Burrows, and A. P. Monkman, Macromolecules 39, 5854 (2006).

[26] M. Knaapila, V. M. Garamus, F. B. Dias, L. Almásy, F. Galbrecht, A. Charas, J. Morgado, H. D. Burrows, U. Scherf, and A. P. Monkman, Macromolecules 39, 6505 (2006).

[27] M. H. Rahman, C.-Y. Chen, S.-C. Liao, H.-L. Chen, C.-S. Tsao, J.-H. Chen, J.-L. Liao, V. A. Ivanov, and S.-A. Chen, Macromolecules 40, 6572 (2007).

[28] L. L. G. Justino, M. L. Ramos, M. Knaapila, A. T. Marques, C. J. Kudla, U. Scherf, L. Almásy, R. Schweins, H. D. Burrows, and A. P. Monkman, Macromolecules 44, 334 (2011).

[29] J.-H. Chen, C.-S. Chang, Y.-X. Chang, C.-Y. Chen, H.-L. Chen, and S.-A. Chen, Macromolecules 42, 1306 (2009).

[30] C.-Y. Chen, C.-S. Chang, S.-W. Huang, J.-H. Chen, H.-L. Chen, C.-I. Su, and S.-A. Chen, Macromolecules 43, 4346 (2010).

[31] M. Knaapila, L. Almásy, V. M. Garamus, M. L. Ramos, L. L. G. Justino, F. Galbrecht, E. Preis, U. Scherf, H. D. Burrows, and A. P. Monkman, Polymer 49, 2033 (2008).

[32] M. Knaapila, F. B. Dias, V. M. Garamus, L. Almásy, M. Torkkeli, K. Leppänen, F. Galbrecht, E. Preis, H. D. Burrows, U. Scherf, and A. P. Monkman, Macromolecules 40, 9398 (2007).

[33] M. Knaapila, R. Stepanyan, M. Torkkeli, V. M. Garamus, F. Galbrecht, B. S. Nehls, E. Preis, U. Scherf, and A. P. Monkman, Phys. Rev. E 77, 051803 (2008).

[34] D. W. Bright, F. B. Dias, F. Galbrecht, U. Scherf, and A. P. Monkman, Adv. Funct. Mater. 19, 67 (2009).
[35] D. W. Bright, F. Galbrecht, U. Scherf, and A. P. Monkman, Macromolecules 43, 7860 (2010).

[36] Q. Ying and B. Chu, Macromolecules 20, 362 (1987).

[37] W. W. Graessley, Polymer 21, 258 (1980).

[38] G. ten Brinke, F. E. Karasz, and W. J. MacKnight, Macromolecules 16, 1827 (1983).

[39] M. Grell, D. D. C. Bradley, G. Ungar, J. Hill, and K. S. Whitehead, Macromolecules 32, 5810 (1999).

[40] D. I. Svergun, J. Appl. Crystallogr. 25, 495 (1992).

[41] H.-G. Haubold, K. Gruenhagen, M. Wagener, H. Jungbluth, H. Heer, A. Pfeil, H. Rongen, G. Brandenberg, R. Moeller, J. Matzerath, P. Hiller, and H. Halling, Rev. Sci. Instrum. 60, 1943 (1989).

[42] P. W. Schmidt, in Modern Aspects of Small-Angle Scattering, edited by H. Brumberger (Kluwer Academic Publishers, The Netherlands, 1995), Chapter 1.

[43] S. Mallam, F. Horkay, A.-M. Hecht, and E. Geissler, Macromolecules 22, 3356 (1989).

[44] M. Ariu, D. G. Lidzey, and D. D. C. Bradley, Synth. Met. 111112, 607 (2000).

[45] M. Surin, E. Hennebicq, C. Ego, D. Marsitzky, A. C. Grimsdale, K. Müllen, J.-L. Brédas, R. Lazzaroni, and P. Leclère, Chem. Mater. 16, 994 (2004).

[46] R. Traiphol, N. Charoenthai, T. Srikhirin, and D. Perahia, Synth. Met. 160, 1318 (2010).

[47] D. M. DeLongchamp, R. J. Kline, Y. Jung, D. S. Germack, E. K. Lin, A. J. Moad, L. J. Richter, M. F. Toney, M. Heeney, and I. McCulloch, ACS Nano 3, 780 (2009).

[48] M. J. Lee, D. Gupta, N. Zhao, M. Heeney, I. McCulloch, and H. Sirringhaus, Adv. Funct. Mater. 21, 932 (2011).

[49] D. Perahia, R. Traiphol, and U. H. F. Bunz, J. Chem. Phys. 117, 1827 (2002).

[50] Y.-C. Li, C.-Y. Chen, Y.-X. Chang, P.-Y. Chuang, J.-H. Chen, H.-L. Chen, C.-S. Hsu, V. A. Ivanov, P. G. Khalatur, and S.-A. Chen, Langmuir 25, 4668 (2009).

[51] W.-C. Ou-Yang, C.-S. Chang, H.-L. Chen, C.-S. Tsao, K.-Y. Peng, S.-A. Chen, and C. C. Han, Phys. Rev. E 72, 031802 (2005).

[52] S.-J. Zhang, L. D. Pfefferle, and C. O. Osuji, Macromolecules 43, 7549 (2010).

[53] R. Abbel, R. van der Weegen, W. Pisula, M. Surin, P. Leclère, R. Lazzaroni, E. W. Meijer, and A. P. H. J. Schenning, Chem. Eur. J. 15, 9737 (2009).

[54] H. Y. Byun, I. J. Chung, H. K. Shim, and C. Y. Kim, Chem. Phys. Lett. 393, 197 (2004). 